\title{
USE OF THE ECHOGRAM IN THE LOCATION AND DIAGNOSIS OF INTRA-OCULAR FOREIGN BODIES*
}

\author{
BY
}

\author{
ARVO OKSALA AND ANTTI LEHTINEN \\ From the Ophthalmic Department of the Central Finland Regional Hospital, \\ Jyväskylä, Finland
}

IT is often difficult to locate an intra-ocular foreign body. Sometimes it may be seen and localized by biomicroscopy, ophthalmoscopy, gonioscopy, or diaphanoscopy. Yet radiological examination is necessary in every case, because the foreign body seen by the foregoing methods is not invariably the only one present in the eye. Although $x$-ray methods (Comberg, Dixon, Goldmann and Bangerter, Sweet, Vogt) are fairly safe, an error of as much as $2 \mathrm{~mm}$. may occur so that in some cases it may be difficult to determine whether a foreign body in the vicinity of the posterior wall lies within the eye or outside it.

Some foreign bodies, such as aluminium slivers, stones, wood chips, and glass splinters, cannot be clearly seen on the $x$-ray film, and ophthalmoscopy is not always helpful. Those foreign bodies, which it has been difficult or impossible to locate, may now be found by means of the echogram registered by ultrasound equipment.

Oksala and Lehtinen (1957) and Baum and Greenwood (1958) found that ultrasonic waves could be used for locating intra-ocular foreign bodies, whether they were visible on the $x$-ray film or not. Oksala (1958) used both radiological and ultrasonic methods in diagnosis.

\section{Equipment}

The ultrasonic apparatus (Fig. 1 $a$, opposite) was constructed by Dr.J. a. H. Krautkrämer, Gesellschaft für Elektrophysik, Cologne. Beside it a silver-plated barium titanate crystal $(c)$ of $5 \mathrm{~mm}$. diameter and $4 \mathrm{Mhz}$ is seen hanging at the end of a cable. The functioning of this equipment has been described by Oksala and Lehtinen (1957) and Oksala (1958).

Fig. $1 b$ (opposite) shows an interferometer constructed by the same firm which is used for measuring the diameter of the eye and its internal parts.

Three of the crystals manufactured for ophthalmological examination in accordance with our instructions are shown in Fig. 2 (opposite). 


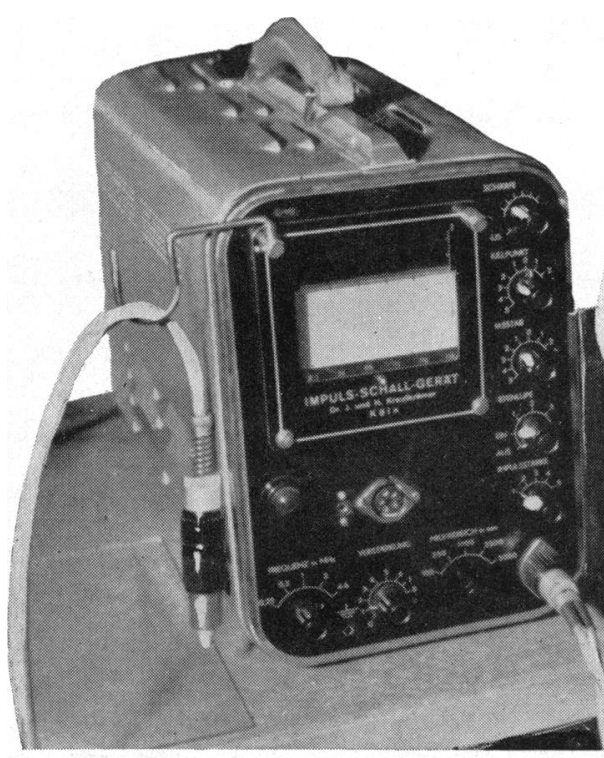

(c)

(a)

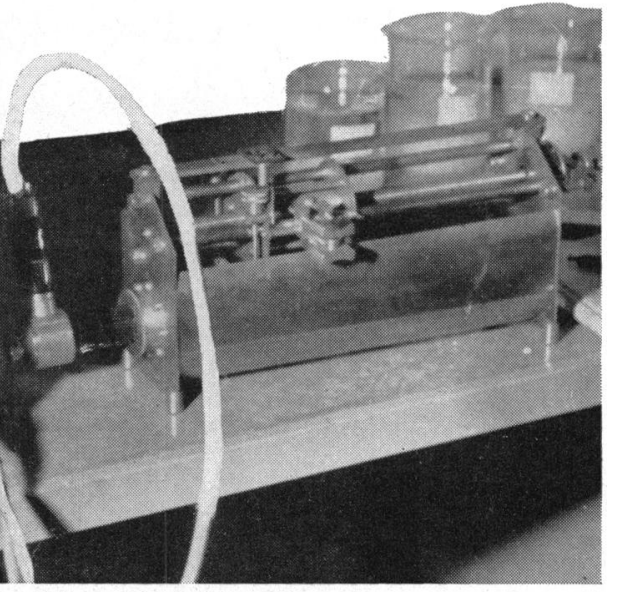

(b)

Fig. 1.-(a) Ultrasound equipment, with a $4 \mathrm{Mhz}$ and $5 \mathrm{~mm}$. crystal (c) hanging beside it at the end of a cable. (b) Interferometer.

Fig. 2.-Three silver-plated barium tit a na te crystals for ophthalmological examination:

The curved handle enables the sonic wave to be directed through the eye from back to front, instead of in the usual direction from front to back.

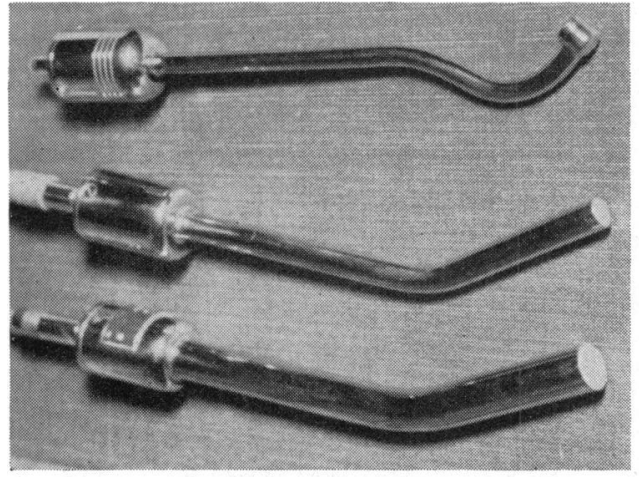

(a) $4 \mathrm{Mhz}$ and $5 \mathrm{~mm}$.

(b) $6 \mathrm{Mhz}$ and $5 \mathrm{~mm}$.

(c) $6 \mathrm{Mhz}$ and $10 \mathrm{~mm}$.

Method of Use

The eye was anaesthetized with 3 per cent. cornecain drops (Hoechst), and a 1 per cent methylcellulose solution was pipetted into it as sound transmitter, which also formed a protective film between the crystal and the eye. The crystal was then pressed very gently against the eye, and the echogram was reflected on the scale of the Braun tube and photographed. While the operation is in progress it is advisable to use a blepharostat.

The near field of all the crystals shown here, i.e. the cylindrical part of the sound impulse, is longer than the diameter of the eye, and this makes it possible to direct the sound waves. By varying the place of contact of the crystal with the eye, an echogram can be obtained from different parts of the eye. 
If the impulse is directed axially through the eye, the normal echoes seen in Fig. 3 are obtained. The figure shows on the left a high initial echo or the initial impulse, which does not find sufficient space on the scale.

FIG. 3.-Normal echogram of the eye when sound waves pass through it axially.

On the left a high initial echo followed by the short zero-line of the anterior chamber. Next the echoes from the anterior and posterior surface of the lens separated by a short zero-line. The long zero-line represents the vitreous. The echoes on the right are emitted by the posterior surface of the eye and the retrobulbar tissues.

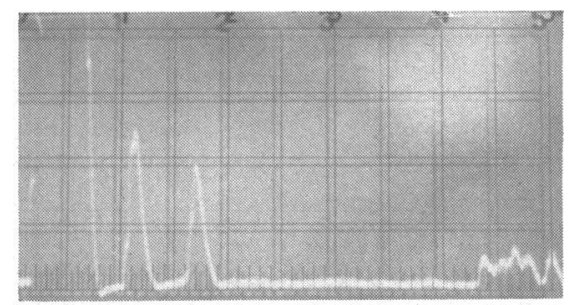

This echogram was obtained when the impulse power of the machine was 3 and the amplification 10. The frequency of the crystal was $4 \mathrm{Mhz}$ and its diameter $5 \mathrm{~mm}$. If the impulse is directed through the sclera into the interior of the eye beyond the lens, only the initial echo will appear on the screen, and then the zeroline of the vitreous and the echo of the posterior wall of the eye. The sound velocity of different parts of the eye has been measured with considerable accuracy (Oksala and Lehtinen, 1958), and the scale on the screen enables one to calculate in millimetres how far the surface which is returning the echo lies from the anterior or posterior wall.

As illustrated by Fig. 3, the initial echo also comprises a so-called silent area, the pathological changes of which are not seen in the echogram, but a foreign body in a position corresponding to the end part of the initial echo will produce a separate peak within the descending line. If the crystals shown in Figs 1 and 2 are used, the length of the silent area will vary, according to the power of the impulse, from 2 to $5 \mathrm{~mm}$.

Since intra-ocular foreign bodies and tumours often lie close to the surface of the eye, in the sclera, chamber angle, or ciliary body, attempts should be made to shorten this silent area as much as possible. By using intermediate pieces of Plexiglass, we were able to reduce the silent area so that the resolving limit was about $1 \mathrm{~mm}$.

Foreign bodies under the surface were always looked for in front of the equator by pressing a crystal fitted with such an intermediate piece against the eye surface. If a foreign body lies below this intermediate piece at $1 \mathrm{~mm}$. distance or more, it will emit an echo peak, which will be seen either in association with the falling part of the initial impulse, or as a separate echo. Superficial foreign bodies behind the equator can be sought without the intermediate piece, by directing the sound wave against them from the opposite part of the eye. Our experience has shown that in these cases also the resolving limit is about $1 \mathrm{~mm}$. A foreign body with an acoustic density markedly different from that of its surroundings will produce either an echo partly incorporated with the posterior wall echo or a separate peak, if it is situated about $1 \mathrm{~mm}$. in front of or behind the sclera.

Fig. 4 (opposite) shows a $4 \mathrm{Mhz}$ and $10 \mathrm{~mm}$. crystal with different Plexiglass pieces which can be joined to it. These vary in length from 9, and 13, to $16.4 \mathrm{~mm}$., with diameters of 12,14 , and $15.5 \mathrm{~mm}$. The pieces are connected to the crystal by means of a connecting socket and a piece of Plexiglass.

Fig. 5 (opposite) shows the crystal fitted with the screw-in. We used oil as the contact substance between the crystal and the extra piece. 


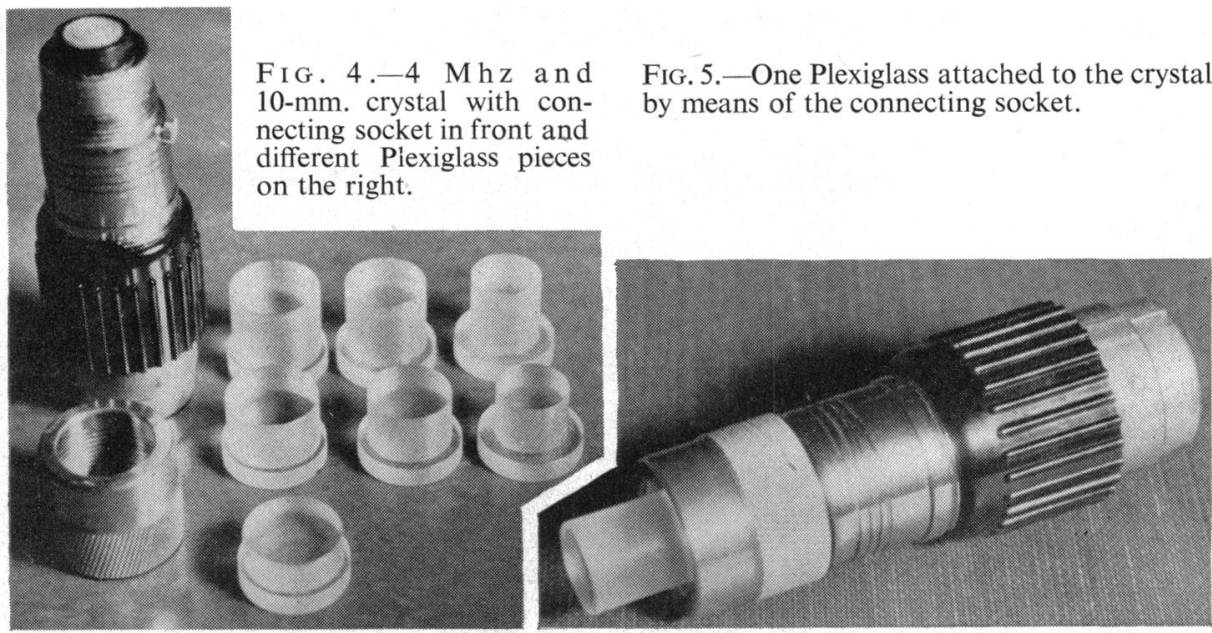

When we used a 9-mm. intermediate piece and directed the sound axially, the echogram seen in Fig. 6A was obtained. The echo marked by an arrow was reflected by the anterior surface of the lens, and the echo following it is a multiple of the echo coming from the Plexiglass and the corneal border surface. In Fig. 6B the zero-point is transferred to the left. When a $13-$ or $16.4-\mathrm{mm}$. Plexiglass is used, ultrasound is so much weakened that it becomes more difficult to obtain an echo from the anterior surface of the lens, but a foreign body with a much greater acoustic density produces a clear echo even from the depth of the posterior lenticular surface. We used these Plexiglass pieces in exploring the area below the surface of the eye to a depth of 4- to 6-mm. Deeper than that a Plexiglass was not used.

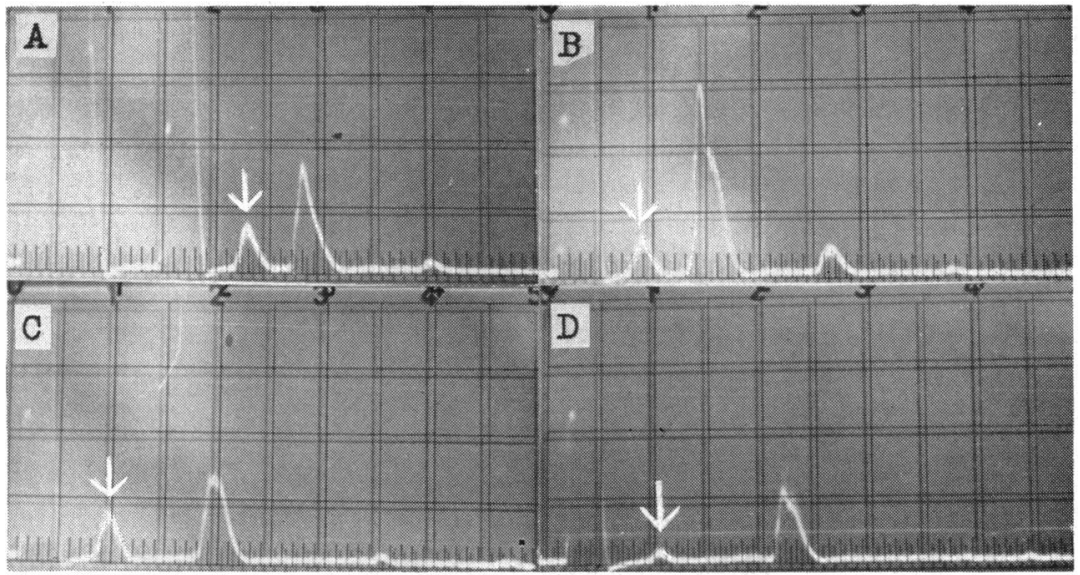

Fig. 6.-Echograms obtained by using Plexiglasses of different lengths. The arrow in each case marks the echo from the anterior surface of the lens and that from the posterior surface is not seen. (A) The initial impulse is seen on the left followed by a high echo from the surface of the Plexiglass and the cornea.

(B, C, and D) The zero point has been moved so far to to the left that the initial echo is out of view and that produced on the border of the Plexiglass and the cornea can now be seen in its place.

In $A$ and $B$ a Plexiglass of $9 \mathrm{~mm}$. was used, in C $13 \mathrm{~mm}$., and in D $16.4 \mathrm{~mm}$. 
The resolving limit of the Plexiglass pieces is also made clear by our observation that they enable us to record the echo emitted by the posterior surface of the bovine cornea. The human cornea is so thin that we failed to obtain a separate echo from its posterior surface.

Besides showing the site of the foreign body the echogram can also give an approximate idea of its size. The longer the foreign body is the longer will be the echo produced by it. For example, the vitreous of a bovine eye with a small wooden particle implanted into it was placed into a cuvette, and the crystal was pressed against the vitreous so that the impulse encountered the particle (Fig. 7A); the screen then showed the echogram seen in Fig. 7B, from which the length of the chip in the axis of the impulse could be computed.

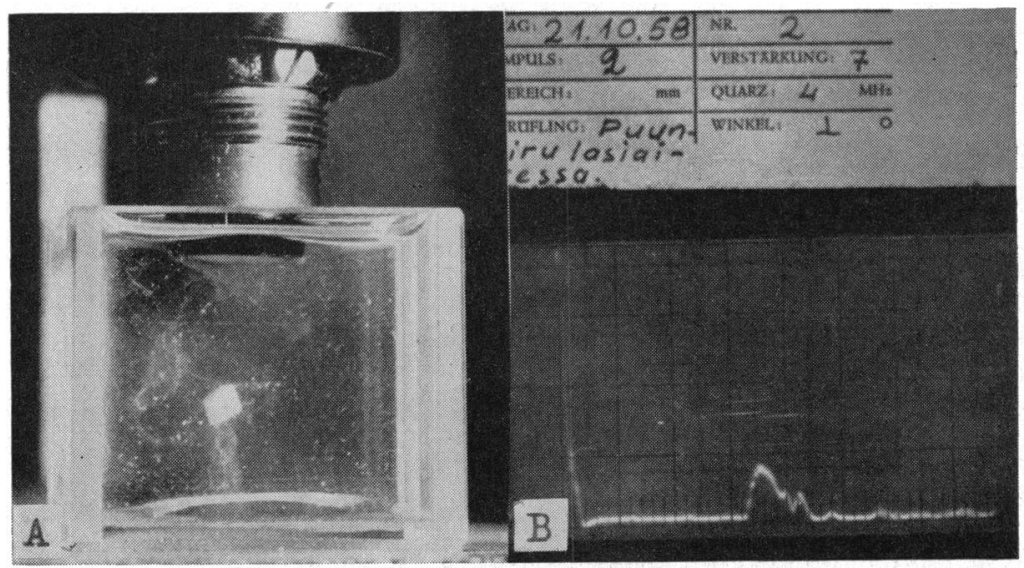

FIG. 7 (A).-Vitreous of a bovine eye in a cuvette, with an implanted wood chip. Above it is the crystal, which is directed towards the chip.
FIG. 7 (B).-Echogram so obtained, the echoes emitted by the wood particle being seen in the middle.

Although the position of a foreign body in the axis of the impulse can be calculated to a millimetre we have not yet succeeded in determining the exact position of the ray within the eye. Superficial foreign bodies in the anterior parts of the eye as far back as the equator can be located with an accuracy of about 1 to $2 \mathrm{~mm}$., but those in the central and posterior parts of the eye are liable to an error of several millimetres, the main difficulty being to find the correct meridian. Our best results were obtained by combined radiological and ultrasonic examinations.

\section{Case Reports}

Case 1, a male aged 26, had had a metal sliver penetrate his left eye while using a hammer, and was admitted to hospital on the same day.

The visual acuity of the left eye was 1.0 and the ocular tension $20 \mathrm{~mm}$. $\mathrm{Hg}$ (Schiøtz). A perforating wound approximately $2 \mathrm{~mm}$. long was seen in the sclera of the left eye close to the limbus on the nasal side. Slight hyphaema was present in the anterior chamber. A small iron sliver could be seen with the ophthalmoscope in the retina below and temporally, and it had seemingly penetrated as far as the sclera. The foreign body was surrounded in the retina by oedema and small haemorrhages. 
The foreign body was located by $x$ rays by Comberg's method and this also indicated that the iron sliver was lying partly in the retina and partly in the sclera. No other foreign bodies were seen.

By the ultrasonic examination normal echograms were obtained from every part of the left eye, except from the direction of the sliver seen in the retina. A crystal of $6 \mathrm{Mhz}$ and $5 \mathrm{~mm}$. was used, force 4, amplification 7. Fig. 8 shows the echogram obtained by directing the sound waves against the iron sliver. On the right the arrow marks the echo partly incorporated with that from the posterior surface of the eye. The echogram showed the head of the sliver to lie about $1 \mathrm{~mm}$. in front of the fundus. The vitreous produced a pure zero line throughout, which showed that its structure was little damaged.

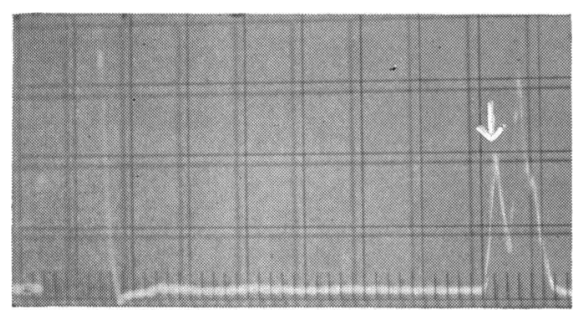

Fig. 8.-Echogram obtained from the left eye in Case 1 when the sound impulse encountered the iron particle embedded in the retina and the sclera (arrow). The echo emitted by the particle is incorporated to some extent with that from the posterior surface of the eye.

The extraction was carried out by first applying diathermy to the sclera at the site of the splinter, and removing the iron particle by means of a magnet through the scleral wound thus made. The eye healed well and the visual acuity remained at 1.0.

Case 2, a male aged 41, had had a metallic particle enter the right eye about some 4 years before while using a hammer. He was unable to remember what metal he was working on at the time, and because the visual acuity was unimpaired and he had no pain in the eye, he had not sought medical aid. Two years later, however, redness and pain began to appear in the right eye from time to time, and in the course of one year there was continued deterioration of vision.

Examination.-The visual acuity of the right eye was $\frac{1}{\infty}$, and the perception of light uncertain. Slight ciliary injection was seen in the eye. The ocular tension was $55 \mathrm{~mm}$. $\mathrm{Hg}$ (Schiøtz). The cornea was oedematous and the scar of a small perforating wound was seen at its temporal margin. There was a corresponding small hole in the iris. The posterior lens capsule showed a central opacity, there were abundant floating opacities in the vitreous, and the fundus could not be seen distinctly. However, the optic disc appeared clearly excavated. No foreign body could be located with the ophthalmoscope, the slit lamp, or $x$ rays.

By the ultrasonic examination normal echograms were obtained everywhere in the eye, except directly from below in the region of the equator. A crystal of $6 \mathrm{Mhz}$ and $5 \mathrm{~mm}$. was used, force 4, amplification 10. By directing the sound wave from above and from behind the limbus down to the equator the echogram seen in Fig. 9 was obtained. Close to the echo from the posterior surface of the eye, a high sharp peak was seen (arrow).

Fig. 9.-Echogram obtained from the right eye in Case 2 when the sound impulse encountered the foreign body in the vitreous (arrow).

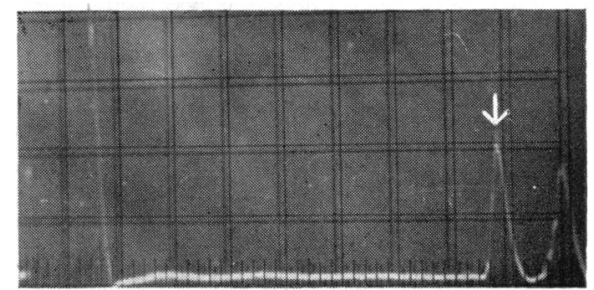


This suggested the presence of a foreign body in the vitreous about $3 \mathrm{~mm}$. in front of the fundus. Low echo peaks could be obtained from the vitreous only in places.

Since pain in the right eye was neither persistent nor strong, the patient has not yet consented to have the eye enucleated, and it is not possible to establish what foreign body was involved, but it is almost certainly a metal fragment which cannot be seen by $x$ rays.

Case 3, a male aged 22, got stone chips in both eyes when a chlorate charge exploded prematurely in a hole drilled into a rock. Stone chips were abundant in the skin of the face and on the surface of the eyes, and some of them were immediately removed, but each eye showed a perforating wound some $2 \mathrm{~mm}$. long, in the right eye on the margin of the cornea, and in the left within the sclera. The lens of the right eye was opaque, so that the deeper parts of the eye could not be examined with the slit lamp or ophthalmoscope. The left eye was hypotonic, and the anterior chamber was full of blood. Visual acuity in both eyes was $\frac{1}{\infty}$ with slight perception of light.

The perforating wounds in both eyes were immediately sutured, and the eyes were treated with atropine drops and subconjunctival penicillin $(500,000$ units $/ 0.5 \mathrm{ml}$. once daily). No infection appeared in the eyes. Radiological examination failed to reveal any foreign bodies in either eyes or orbits.

By ultrasonic examination, however, it proved possible to find the stone chips which had penetrated into the eyes. The crystal was the same in Case 2, force 5, amplification 10.

Fig. 10 shows the echogram from the right eye when the sound waves had encountered the stone chip. It was calculated that the chip was situated low down in the vitreous, between the equator and the ora serrata and about $4 \mathrm{~mm}$. in front of the fundus. The vitreous reflected only a zero line in the echogram.

Fig. 11 represents the echogram of the left eye after the sound waves had encountered the stone chip. It was calculated that a large stone chip about $2.5 \mathrm{~mm}$. long was lying low down in the vitreous immediately behind the lens about $9 \mathrm{~mm}$. from the surface of the eye. The vitreous emitted numerous low echoes in every direction which indicated the presence of vitreous opacities.

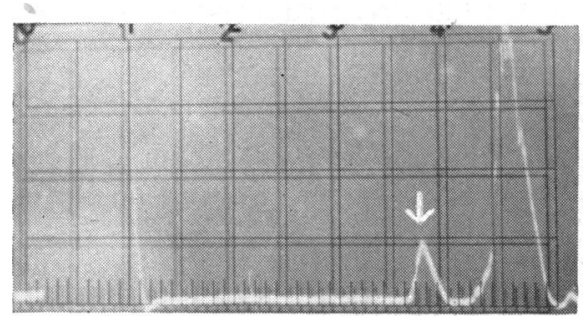

FIG. 10.-Echogram obtained from the right eye in Case 3 when the sound impulse encountered the stone particle situated in the vitreous (arrow).

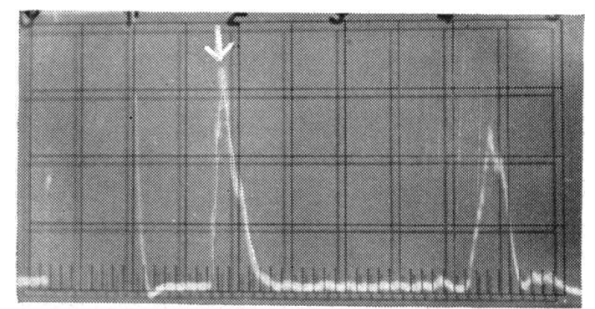

Fig. 11.-Echogram obtained from the left eye in Case 3 when the sound impulse encountered the stone particle in the anterior part of the eye (arrow).

Operation.-Linear cataract extraction was performed on the right eye. No vitreous was lost and post-operatively the visual acuity was 0.5 with glasses. A small stone chip could now be seen with the ophthalmoscope in the lower part of the vitreous. Since the operation the eye has remained symptom-free and the patient is troubled only by slight photophobia. Post-operatively the eye was treated with atropine and prednisolone drops. No removal of the stone chip from this, the patient's only seeing eye, has been contemplated. 
The left eye became completely blind 2 weeks after the accident with persistent hypotony. The cornea was much injected so that the interior of the eye could not be seen. Nevertheless, the eye has remained fairly quiet and treatment has been continued with local atropine and prednisolone drops.

\section{Discussion}

The echogram of the eye registered by ultrasonic equipment has proved very useful in diagnosing intra-ocular foreign bodies. At present both radiological and ultrasonic methods are used. With the aid of Plexiglass the echogram will record those which are as close as about $1 \mathrm{~mm}$. to the surface of the eye, and in such cases the ultrasonic method is as accurate as the $x$-ray method. When the foreign body is very deep in the eye $x$ rays are still found to be more accurate.

The ultrasonic method has the great advantage of being quickly and easily applicable while the operation is in progress, and also shows to what extent the intra-ocular structures have been damaged by indicating the presence of vitreous haemorrhage, retinal detachment, and luxation of the lens. The length of the foreign body in the direction of the impulse can also be roughly calculated from an echogram. By directing impulses in several directions an approximate idea of the size and shape of the foreign body can be obtained.

If a foreign body is invisible to $x$ rays and to ophthalmoscopy, ultrasonic examination is the only method of locating it (Cases 2 and 3). The shape of an echo peak emitted by a wood, glass, stone, or aluminium particle is sufficient to differentiate it from that emitted by a detached retina. Luxation of the lens produces two echo peaks separated from each other by a zero line approximately $4 \mathrm{~mm}$. long. Vitreous haemorrhages and opacities do not cause any diagnostic difficulties in this respect.

Case 1 is an example of the way in which ultrasonic examination reveals whether the foreign body in the vicinity of the posterior surface of the eye is lying inside the eye, in the sclera, or outside the eye.

Finally, this method of investigation is quite safe and has no cumulative effect such as may arise from frequent use of $x$ rays.

\section{Summary}

The ultrasonic echogram records the position of foreign bodies which produce no shadow on a $x$-ray film. Examination by ultrasound can be carried out easily and quickly in the course of an operation, and may also serve to disclose such pathological conditions as retinal detachment, luxation of the lens, and vitreous haemorrhage.

By using intermediate Plexiglass pieces, it is possible to distinguish even those foreign bodies which lie only $1 \mathrm{~mm}$. from the surface of the eye. This 
method is as accurate as $x$ rays in locating foreign bodies in the anterior part of the eye and close to its surface, but $x$ rays are more accurate for locating foreign bodies in deeper sites.

The authors describe their research equipment and method of using it, and report three cases which demonstrate its use.

\section{REFERENCES}

Baum, G., and Greenwood, I. (1958). A.M.A. Arch. Ophthal., 60, 263.

OKSAlA, A. (1958). Nord. Med., 59, 721.

(1959). Klin. Mbl. Augenheilk., (in the press)

and Lehtinen, A. (1957). Ophthalmologica (Basel), 134, 387.

(1958). Acta ophthal. (Kbh.), 36, 633. 